\title{
Alloconfrontations réflexives par vidéoformation : Analyse de l'utilisation de la plateforme Néopass@ction par des formateurs et des professeurs des écoles stagiaires
}

\section{Serge Leblanc et Céline Blanes Maestre}

\section{(2) OpenEdition \\ Journals}

Édition électronique

URL : http://journals.openedition.org/trema/3432

DOI : $10.4000 /$ trema.3432

ISSN : 2107-0997

Éditeur

Faculté d'Éducation de l'université de Montpellier

Édition imprimée

Date de publication : 1 mai 2016

Pagination : 115-127

ISBN : 979-10-96627-00-4

ISSN : $1167-315 X$

\section{Référence électronique}

Serge Leblanc et Céline Blanes Maestre, «Alloconfrontations réflexives par vidéoformation : Analyse de l'utilisation de la plateforme Néopass@ction par des formateurs et des professeurs des écoles stagiaires », Tréma [En ligne], 44 | 2016, mis en ligne le 01 juin 2016, consulté le 04 mai 2019. URL: http://journals.openedition.org/trema/3432 ; DOI : 10.4000/trema.3432 


\title{
Alloconfrontations réflexives par vidéoformation : Analyse de l'utilisation de la plateforme Néopass@ction par des formateurs et des professeurs des écoles stagiaires
}

\author{
Serge Leblanc et Céline Blanes Maestre
}

\section{Introduction}

1 Les milieux professionnels, les responsables de formation et les décideurs sont de plus en plus nombreux à s'interroger sur l'élaboration et l'utilisation d'environnements simulateurs ${ }^{1}$ du travail (Durand, 2008 ; Pastre, 2005) dans les cursus de formation initiale ou continue et sur le développement de modalités de formation hybride. Les enjeux sont à la fois d'optimiser les dispositifs de formation tout en minimisant les coûts. Dans le domaine de la formation des enseignants, ces environnements se concrétisent surtout par des dispositifs accessibles à distance via des plateformes en ligne qui proposent de visionner des films de classe et d'entretiens avec des enseignants et/ou d'autres acteurs (chefs d'établissement, inspecteurs, chercheurs...).

2 Au niveau international, de nombreuses études (voir par exemple la note de synthèse de Gaudin et Chalies, 2012) ont montré que ces dispositifs contribuaient aussi bien au développement des compétences disciplinaires spécifiques (par exemple pour l'enseignement de l'éducation physique: Prusak, Graham et Graser, 2010; pour l'enseignement des mathématiques: Santagata, 2009) qu'aux compétences professionnelles transversales (Brunvand et Fishman, 2006; Ria et Leblanc, 2012 ; Seidel, 
Blomberg et Renkl, 2013). Au sein de la formation initiale des enseignants où elle est très utilisée (Calandra et Puvirajah, 2011) mais aussi en formation continue (Van Es et Sherin, 2008), les études ont pointé les effets bénéfiques en termes de renforcement de l'intérêt pour le métier d'enseignant, d'analyse réflexive indexée à des pratiques réelles, et de développement professionnel notamment par une meilleure prise en compte de l'activité des élèves.

Il ressort également de l'état de l'art deux recommandations importantes pour favoriser ces effets bénéfiques: la nécessité de présenter des vidéos ${ }^{2}$ en adéquation avec les capacités et besoins des personnes en formation, l'utilité de les accompagner lors du visionnage de vidéos. Qu'en est-il de ces effets lorsque ce type d'environnement est utilisé de manière autodidacte ou dans des dispositifs totalement à distance ? Cette contribution vise à apporter des éléments de réflexion en s'appuyant sur l'analyse de deux études de cas distinctes réalisées à partir de l'utilisation de visionnage de vidéos issues de la plateforme Néopass@ction, l'une, avec des professeurs des écoles stagiaires (PES) en position d'autodidacte, et l'autre, avec un groupe de formateurs interagissant dans un dispositif totalement à distance.

4 La plateforme Néopass@ction a été conçue dans la perspective de confronter les enseignants débutants dans le métier à des problèmes professionnels typiques auxquels ils risquent rapidement être exposés. Concrètement, elle propose, au plan national, des référentiels partagés ${ }^{3}$ sur le travail réel des enseignants comme ressources potentielles pour les débutants en autoformation ou en formation institutionnalisée en présentiel ou à distance avec un formateur universitaire ou de terrain (Ria et Leblanc, 2011). En se situant en rupture par rapport à une approche prescriptive qui viserait à faire construire des connaissances pédagogiques en visionnant les «bonnes pratiques» d'enseignants experts, elle cherche à favoriser la création de liens entre différentes expériences, la sienne et celles des autres, celles des novices et des plus expérimentés, en s'appuyant prioritairement sur une déconstruction-reconstruction des expériences typiques des enseignants débutants. Des études récentes analysant l'activité de visionnage de vidéo issues de cette plateforme par des enseignants en formation ont montré des effets de réassurance et de déculpabilisation, de reconnaissance de soi à travers les pratiques de pairs, d'autoévaluation et d'auto-prescriptions ajustées à leurs difficultés professionnelles du moment (Flandin, Leblanc et Muller, 2015). À travers nos deux études, qui ont pour caractéristique commune, le fait de visionner des vidéos de manière solitaire, nous avons étudié les effets de ces explorations pour les uns totalement laissées à leurs initiatives (PES) et pour les autres très cadrées et débouchant sur une production à réaliser (formateurs).

\section{Contextualisation des deux études et méthodes}

La première étude a consisté à faire revivre une séance d'autoformation ${ }^{4}$ sur la plateforme Néopass@ction avec deux PES en se rapprochant le plus possible des conditions de fréquentation en autodidacte, à savoir qu'aucune prescription extérieure en termes de temps, de lieu de travail ou de tâche quelconque ne leur a été formulée. L'objectif était d'étudier cette activité peu accessible en raison de son caractère privé mais pourtant intéressante à explorer au vu du développement des actes d'autoformation sur hypermédias. L'activité des deux PES, engagées dans une séance de navigation libre, solitaire et autonome sur la plateforme, a été étudiée à partir des enregistrements filmés 
de ces explorations et d'un entretien d'autoconfrontation avec chacune d'elles les amenant à commenter, raconter leur expérience vécue instant après instant de façon à en restituer la signification dynamique sous-jacente.

6 La seconde étude a consisté à analyser les traces d'activités produites à partir de la plateforme Néopass@ction et déposées sur une autre plateforme en ligne (Dokéos) par 17 formateurs (trices) suivant une unité de formation totalement à distance qui portait sur l'analyse de l'activité. Les objectifs de cette formation visaient à leur faire à la fois vivre une expérience d'enseignement à distance afin d'en percevoir les intérêts et limites et en même temps découvrir et exploiter les ressources vidéo de la plateforme Néopass@ction (cf. figure 1) dans une perspective d'aide des enseignants débutants à analyser leur pratique professionnelle. Pour cela, un parcours en 5 étapes très cadré leur était proposé : a) commenter le visionnage de deux vidéos de classe contrastées, b) identifier les éléments organisateurs de l'activité des enseignants à partir des entretiens "vécu professionnel », c) repérer les apports de ces entretiens à la compréhension de l'activité, d) envisager des axes de réflexion exploitables en formation d'enseignants novices, et e) participer à un forum sur les intérêts-limites de l'exploitation de la plateforme en formation. L'objectif de la recherche était de repérer les engagements des formateurs dans ce nouvel environnement et d'apprécier leur niveau d'appropriation de ces ressources ainsi que les difficultés rencontrées.

Figure 1 : Schématisation de l'espace «Vidéos »de Néopass@ction

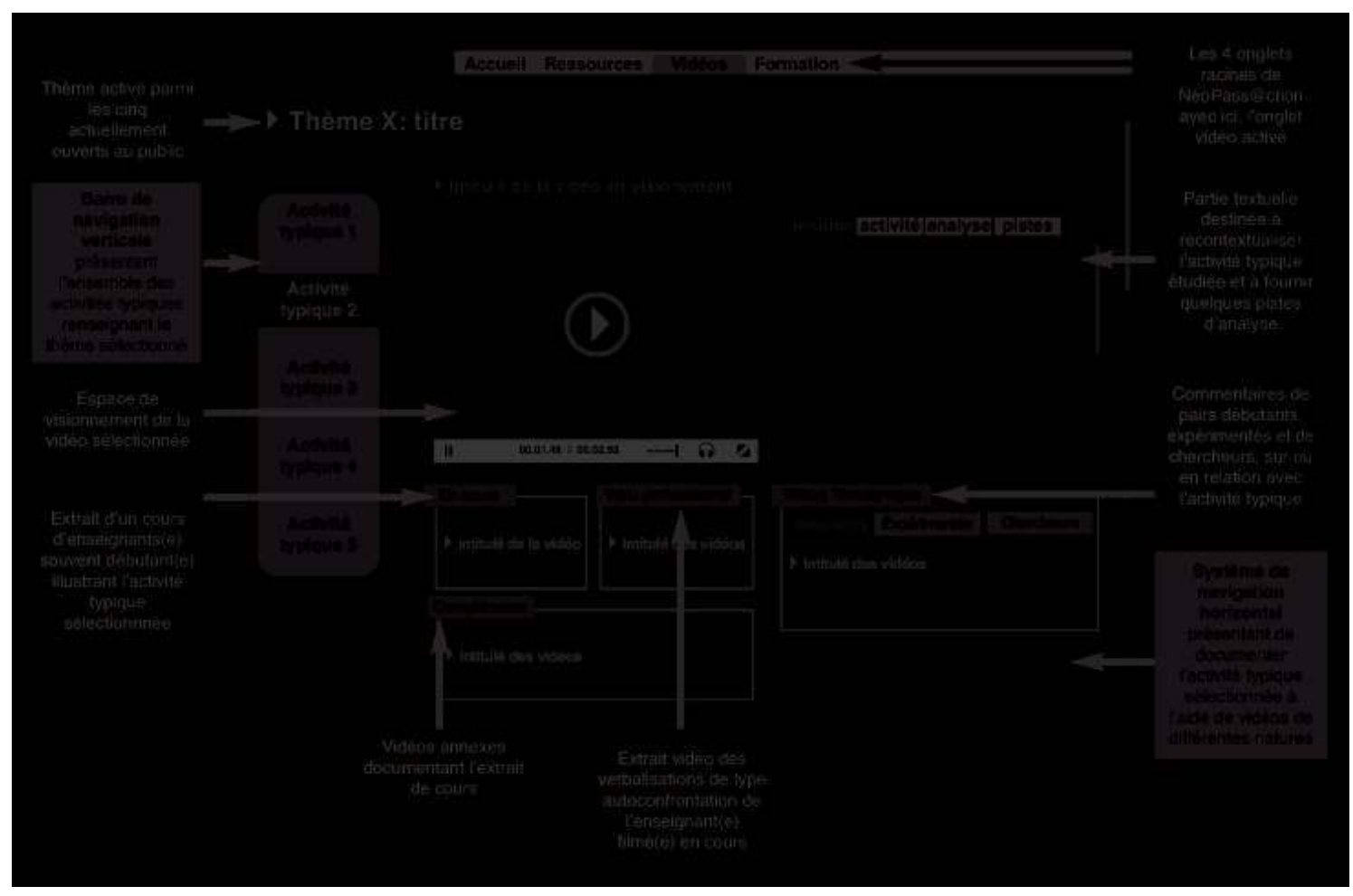

\section{Résultats}

7 La diversité des données recueillies lors de ces deux études permet d'éclairer sous différents angles les trois axes d'analyse suivants : le premier s'intéresse à la question de l'appropriation de la plateforme en tant que nouvel espace de travail, le deuxième met à 
jour les différentes postures adoptées par les PES et les formateurs (trices) face à la mise en réflexion sur le métier enseignant suscitée par le contenu de la plateforme, et le troisième interroge la distanciation qui s'opère entre l'activité des personnes navigant et celles des personnes à l'écran.

\section{III.1. L'appropriation d'un nouvel espace virtuel de travail : dynamiques, potentialités et limites}

8 Que cette plateforme soit utilisée en autoformation proche de l'autodidaxie, comme c'est le cas ici pour les PES, ou en autoformation davantage " éducative " (Carre, Moisan et Poisson, 1997) comme pour les formateurs, les données révèlent l'existence d'un temps et de modalités d'appropriation particuliers. Ces résultats nous paraissent intéressants pour la conception d'environnement de formation, de parcours de formation ou d'accompagnement des apprenants.

\section{III.1.1. Du point de vue des PES}

9 La mise en parallèle de l'activité silencieuse $\mathrm{f}^{5}$ des deux PES à partir de leurs verbalisations en autoconfrontation simple (Theureau, 2004), nous a permis d'identifier leurs dynamiques d'appropriation de l'environnement de formation. L'appropriation est ici appréhendée en tant que phénomène. Lors de leur séance en autoformation, les premières minutes sur la plateforme s'accompagnent chez les deux PES d'une perte de sens et de repères face au design singulier de la page " Vidéos » (Cf. figure 1) de la plateforme. Clothilde commente cette expérience par: "Je suis restée vraiment longtemps perdue [...] je ne savais pas du tout où aller ! ». Ce premier obstacle se traduit à cet instant par l'incapacité des deux PES à interpréter la structure informationnelle de la page pour en extraire le " potentiel d'action proposé » (Paquelin, 2009). Toutefois, plutôt que de les décourager, ce phénomène conduit chacune d'elles à ouvrir leur focale attentionnelle "Alors je cherche, je lis tout, je re-regarde...». Ici la PES, par l'intermédiaire de son attention élargie, parvient ici à interpréter l'objet « Thème » : « là j'ai compris les thèmes, donc j'suis allée directement vers le thème qui m'intéressait ». Cette attribution de sens restaure alors le dialogue entre ses préoccupations personnelles et ce que lui donne à voir la plateforme. Elle retrouve alors son pouvoir d'agir, qui s'exprime ici par la sélection du thème qui l'intéresse, qui va «le mieux répondre à [son] besoin ", parmi les cinq autres disponibles.

10 L'appropriation de la plateforme s'opère ainsi au fil de la séance par la rencontre entre les éléments intrinsèques aux deux PES tels que l'âge de leurs propres élèves, leurs difficultés professionnelles et actuelles d'ordre pédagogique, didactique, disciplinaire, exprimées sous forme d'intérêts, de préoccupations, et les éléments extrinsèques, ceux de la plateforme, composés des indices perceptifs (donc nécessairement significatifs pour elles) notamment les titres des vidéos, le niveau des élèves dans les vidéos, la période de l'année durant laquelle se déroule la séance filmée, etc. Se construit alors progressivement l'orientation de leur navigation libre au gré de leur découverte et de leur familiarisation concomitante de la plateforme et des vidéos. Dès lors, deux cas de figure sont apparus.

11 Tout d'abord, en regardant la vidéo d'une enseignante débutante confrontée à des difficultés de gestion de l'hétérogénéité, Clothilde commente: "c'est des enfants qui ressemblent plus, à mes élèves, donc euh... j'arrive mieux à me projeter, avec ces p'tits, cet âge-là 
disons». La proximité situationnelle entre cette activité filmée et l'expérience professionnelle de Clothilde s'opérant tant par l'âge des élèves que par la thématique abordée conduit à une immersion mimétique de la PES repérable à l'expression «je me revois en classe ». Cette proximité favorable à la mise en écho avec sa propre pratique est de nature à soutenir l'engagement de la PES qui maintiendra l'orientation de sa navigation via la mise en place d'un processus d'enquête. En effet, elle prolongera sa navigation en cherchant à documenter davantage cette situation qui fait sens à l'aide du vécu professionnel de l'enseignante filmée (relaté en entretien) et de commentaires de pairs expérimentés.

12 À l'inverse, lorsque les potentialités perçues des éléments extrinsèques (de l'environnement de formation) ne rencontrent pas les préoccupations des PES, l'exploration s'achève et la navigation se réoriente vers une autre voie: Emilie découvrant l'activité typique "Afficher les productions » du thème «Faire parler les élèves à l'élémentaire» commente: «le titre des vidéos me parle pas plus que ça [...] je lis le résumé [...] J'me suis dit que ça répondrait pas forcément à mes problèmes [...] j'essaie de chercher des situations dans lesquelles j'm'identifie, des trucs qui vont me poser problème». Emilie ne visionnera aucune des vidéos rattachées à cette activité typique et se rendra sur une autre. Ainsi, sans arrimage de son propre vécu aux vidéos consultables, la dynamique exploratoire s'essouffle et ne suffit pas à faire perdurer l'orientation de la navigation libre, il est nécessaire que la personne se projette, se reconnaisse dans le contenu, qu'elle y trouve un intérêt, pour entamer un processus d'enquête dont nous postulons qu'il est le signe d'un véritable investissement dans l'acte de formation, porteur par extension d'apprentissage. La dynamique d'appropriation est ainsi composée de deux modes: l'exploration d'une part, qui permet de délimiter le cadre de l'action mais également l'enracinement de sorte que «dans un produit interactif, le sujet devient tour à tour architecte [de son environnement de formation] et habitant » (Paquelin, 2009, p. 162), transformant la plateforme « d'espace comme étendue à celui d'espace habité » (Ibid., p. 164).

\section{III.1.2. Du point de vue des formateurs}

13 Nous avons évalué l'appropriation de l'outil par les formateurs (Néopass@ction et la plateforme Dokéos) à partir de leur production individuelle écrite et des principaux sujets de discussion qui se sont construits dans le forum réalisé en fin de parcours. Sur ce forum intitulé «intérêts-limites de Néopass et de la vidéo formation»: $49 \%$ des propos des formateurs ont traité directement ce sujet ; $17,90 \%$ ont restitué des expériences en écho d'enseignants-formateurs à ce qu'ils ont visionné et/ou se sont projetés dans une future activité de formation ; 17, $85 \%$ ont porté sur des questions-réponses et des points de vue génériques sur la formation ; 11, $80 \%$ ont abordé les difficultés techniques rencontrées pour réaliser les travaux demandés et $4,45 \%$ ont traité de sujets divers. Nous interprétons cette répartition comme la nécessité de prendre en compte trois préoccupations principales : satisfaire aux attentes du formateur en s'engageant dans la tâche demandée, avoir un retour sur sa propre pratique de formateur en redéfinissant la tâche, s'approprier les artefacts. On retrouve la nécessité "d'habiter » l'espace, de le mettre à sa main pour mieux pouvoir explorer l'étendu des possibles.

14 L'évaluation qualitative de l'appropriation de la plateforme vidéo a été repérée à travers les items qui sont ressortis comme étant intéressants du point de vue de la formation professionnelle des enseignants, révélant les potentialités de l'outil : la réassurance et le sentiment d'être membre d'une communauté, le repérage des effets produits de certains 
gestes professionnels sur les élèves, la parole donnée aux enseignants eux-mêmes, la possibilité de juger l'action sans juger celui qui la fait, la projection concrète dans une situation de classe, l'élaboration de perspectives de progrès réalistes et atteignables, la perception des difficultés et des réussites des collègues avec des façons différentes d'agir... Ces éléments de réflexion ont été construits au fil des différentes étapes du parcours proposé comme l'illustrent les propos de cette formatrice en réponse à la question - apports de l'entretien de type « vécu professionnel »- : «Ces entretiens avec les enseignants nous renseignent à la fois sur leurs logiques profondes, leur activité et leurs préoccupations réelles et en acte, les représentations qu'ils se sont forgées et comment ils les font évoluer ». Les visionnages imposés, suivis d'un travail d'interprétation de ce qui se jouait dans les films en intégrant progressivement le point de vue des enseignants ont contribué à la construction d'une vision très favorable de ce type d'environnement comme l'évoque cette collègue comparativement à d'autres dispositifs de formation vécus :

"Les échanges avec nos formateurs et entre stagiaires étaient souvent frustrants car personne n'avait partagé les mêmes situations ni se trouvait avec les mêmes problématiques. Notre questionnement était dicté par l'urgence des cours à préparer. L'absence de réponses quant à : L'entrée en classe? La mise au travail ? L'attitude à adopter face à un incident ? Les rituels? nous faisait vivre notre année de formation comme une mise à l'épreuve. »

En parallèle d'une vision très positive de ce type d'environnement, un certain nombre de limites pointées par les formateurs révèlent tout autant leur niveau d'appropriation de la plateforme du point de vue de son utilité et utilisabilité lorsqu'il se projette dans le cadre d'une formation d'enseignants débutants : le nécessaire accompagnement de l'enseignant pour faire émerger des pistes de remédiation; le regard bienveillant à adopter par rapport aux collègues exposés dans les vidéos; le risque de plaquer un modèle, seul, derrière son ordinateur ; le caractère déstabilisant de certaines vidéos ; la couverture trop réduite des contextes d'enseignement. Compte tenu du caractère très guidé du parcours, peu de difficultés sont apparues lors de la navigation sur la plateforme vidéo. Par contre, des difficultés d'appropriation technique se sont manifestées en rapport avec l'utilisation de Dokéos. Tâtonnement, sensation d'isolement-présence, échecs-apprentissages, impression d'être débutant, découragement-encouragement sont les principaux états évoqués entre eux dans le forum.

\section{III.2. La mise en réflexion du métier en formation : entre résistance et acceptation}

16 Face à la complexité et aux enjeux du métier enseignant, il est fréquent que les débutants sollicitent une formation de type "boite à outils» supposée pallier les urgences de la classe. Plutôt qu'applicationniste, les fondements épistémologiques constitutifs de la plateforme Néopass@ction, la définissent davantage comme étant un «espace d'actions encouragées » (Durand, 2008) où les bonnes pratiques laissent potentiellement place à la problématisation du métier. Dans cette perspective, nous analysons ici les positionnements et apprentissages que suscite ce type d'environnement chez les deux PES puis chez les formateurs, selon qu'elle est acceptée ou non. 


\section{III.2.1. Du point de vue des PES} proche de son vécu en classe pour en extraire des outils. Or, plutôt qu'une bonne pratique c'est une question professionnelle inédite que la vidéo lui renvoie en écho. Il y a, à cet instant, inadéquation entre les attentes initiales de la PES et ce que lui donne à voir la vidéo. De cette inadéquation nait une tension a priori récurrente, identifiable au «mais euh... c'est toujours pareil en fait ». Emilie va alors mettre en balance l'intérêt qu'elle perçoit de poser cette question et l'absence de "bonnes réponses" qu'elle retire de son visionnement. Il semble qu'elle attribue alors cette non-réponse à une règle de métier, ce qui lui permet de faire évoluer cette tension vers l'acceptation de cet état de fait. Cela lui permet de maintenir son engagement dans l'acte de formation, et par un jeu de mimésis, de reprendre à son compte la démarche réflexive des trois enseignants filmés et de se l'approprier.

21 Dans ce second extrait Clothilde, également engagée dans un processus d'enquête vit cette tension tout autrement. Bien que sa recherche de pistes pour sa propre classe s'avère infructueuse, elle retire de son visionnement une mise en réflexion autour de savoirs de métier et savoirs en actes issus des vidéos qu'elle pondère, compare à sa propre pratique et évalue au fil de son explicitation. Pourtant à l'issue de cette phase voici ce qu'elle en dit : "Non, ça ne m'apporte rien [long silence]. Si à part de la réflexion, sur moi, ma pratique, euh, voilà on compare, on réfléchit mais, sinon ça ne m'apporte rien de concret ». Elle se dira déçue et frustrée tout au long de sa navigation ainsi qu'à son issue.

Cette plateforme, comme d'autres environnements numériques actuels, vise à développer chez les personnes en formation « des capacités à maîtriser un agir autonomisant » (Paquelin, 2009, p.170). Dans ce sens, il est implicitement demandé à l'acteur d'opérer, un basculement de la traditionnelle posture de formé à qui l'on fournit de recettes à appliquer, à celle d'individu se formant où la problématisation, la réflexivité, et parallèlement la responsabilité prévalent sur d'hypothétiques bonnes pratiques. Or, comme nous venons de le voir ce basculement pour majeur qu'il soit, ne se décrète pas 
mais s'accompagne et se construit au sein d'un « environnement capacitant » (Falzon, 2013) ${ }^{6}$

\section{III.2.2. Du point de vue des formateurs}

23 Côté formateurs, la problématisation du métier induite par leur formation s'est traduite par la remise en question de cet objet central de leur activité qu'est la définition du «bon enseignant ». Cette réflexion apparait chez eux tout d'abord, indirectement, à travers la réalisation de la tâche de comparaison de l'activité de deux enseignants (un débutant et l'autre plus expérimenté) et ensuite, directement, lors d'un échange spontané sur le forum entre plusieurs formateurs.

La comparaison de l'activité des deux vidéos d'enseignants (débutant et expérimenté) met à jour chez les formateurs, en creux, leur vision de ce que doit être un «bon enseignant ». Ainsi à travers leurs productions écrites individuelles, deux tendances assez contrastées peuvent se manifester. Les propos de ces deux formatrices illustrent une vision différente de la construction de la professionnalité enseignante : la première décrit et commente l'activité de l'enseignant débutant en utilisant systématiquement des tournures de phrases négatives « Il ne paraît pas avoir de solution didactique... cela n'apparaît pas... il ne marque pas son autorité par un seuil... le prof ne crée pas...» ou en portant des jugements critiques «Le mauvais positionnement spatial du prof alimente la distance avec les élèves qui ne reconnaissent pas l'autorité, ni l'accueil », la deuxième formatrice cherche à rendre compte de ce qui organise sa façon de faire «L'entrée en cours de ce jeune enseignant semble marquée par une représentation traditionnelle voire idéalisée dans laquelle les élèves respectent un protocole attendant que l'enseignant veuille bien "tenir son rôle " " : leur transmettre le savoir». La première description-analyse laisse entrevoir qu'il y aurait de bonnes manières de faire à mettre en œuvre alors que la deuxième débouche sur une interrogation: "Le "rôle" de l'enseignant commencerait-il avant la "transmission des savoirs" $»$ ?

De manière complémentaire, une réflexion sur cette thématique s'est instaurée de manière plus directe sur le forum comme l'illustrent les questions échangées : «Un bon enseignant est-il toujours un praticien réfléchi? »; "Qu'est-ce qu'un bon enseignant ?" Est-ce un enseignant qui fait des séances "tip-top" ? Est-ce un enseignant qui se "plante" mais qui est capable d'expliquer pourquoi et comment? Est-ce un enseignant qui ose? Et alors qu'est-ce qu'oser d'un point de vue pédagogique? "; "Qu'en est-il si l'enseignant ne "progresse" pas? N'y a-t-il pas alors un risque de démotivation? N'a-t-on pas aussi l'impression qu'on applique des recettes?»

Si les formateurs n'ont pas apporté de réponses précises à toutes ces questions, il ressort majoritairement de leurs échanges sur le forum que la visée de la formation la plus partagée est de contribuer au développement d'un enseignant réflexif capable de s'autonomiser et de s'émanciper comme le synthétise assez clairement les propos suivants : "La finalité de ces vidéos et autoconfrontations n'est pas de proposer une modélisation mais de tendre à une "méta" réflexion. Il n'existe pas de méthodes toutes faites, cette plateforme aidera un jeune enseignant à construire des représentations, à trouver des réponses, à faire des tentatives d'analyse, à adopter une posture adéquate ». Il ressort de cette analyse que les formateurs partagent à la grande majorité une conception en termes d'aide plutôt que de prothèses cognitives (Rabardel, 1995) où l'environnement de formation propose des " ressources" accompagnant les acteurs dans leurs interprétations et décisions, leur activité étant considérée au cœur de l'efficacité et de la fiabilité. Dans cette perspective 
d'instruments de type "anthropocentrique », il s'agit de rendre les acteurs capables de traiter les inévitables événements imprévus en augmentant leur adaptabilité face aux problèmes qui peuvent survenir dans leur pratique professionnelle.

\section{III.3. De la distance à la réflexivité}

27 Les phénomènes d'échoïsation entre la vidéo de formation et la personne en formation sont à l'origine d'un jeu de simulation produisant d'une part la reviviscence de situations antérieures, d'autre part, l'anticipation des situations nouvelles imaginées. Les chercheurs postulent que ce jeu est constitutif d'un « remodelage de l'expérience » des personnes (Leblanc et Seve, 2012) grâce, notamment, à un processus de « déconstructionreconstruction » (Lussi Borer et Muller, 2015) de leur activité.

Nos données mettent en avant la relation étroite qui se noue entre ce potentiel formatif et la distance qui s'instaure entre un soi, celui des personnes en formation, et un autre, perçu à l'écran.

\section{III.3.1. Du point de vue des PES}

Les résultats présentés précédemment révèlent l'existence un premier degré de distance manifesté par la quête initiale et spontanée de proximité qu'Emile et Clothilde recherchent à travers des «situations dans lesquelles [elles s']identifie[nt] ». Cette proximité est indispensable à leur engagement initial dans l'acte de formation, notamment lors du visionnement de l'activité en classe. La reconnaissance de soi dans l'activité de l'autre est à l'origine d'une mise en dialogue entre activité filmée et activité renvoyée en écho, comme en témoigne Emilie: «Là j'me suis rendue compte qu'ils [les élèves filmés en cours] étaient très autonomes [elle revisionne un bout de la vidéo pour voir la date de la séance écrite au tableau]. Bon, et là j'avoue, j'vois "février" et j'me dis "Bon, ça va c'est normal" [...] par rapport à ce que je vois moi en classe. Ben parce que c'est vrai que c'est assez difficile, des classes, une fois qu'on a la nôtre on ne voit plus que la nôtre, c'est un peu difficile de se situer ». Ici la PES attribue une valeur étalon à l'activité filmée à l'aune de laquelle elle jauge la " normalité » de sa propre situation et s'en trouve rassurée. Lorsqu'un processus d'enquête s'en suit, la recherche d'une majoration, conduit à l'augmentation du degré de distance. Le potentiel formatif de cette phase, parce qu'il situe l'individu dans sa zone proximale de développement, mérite une attention particulière. Clothilde immédiatement après le visionnement de l'activité et des autoconfrontations d'un pair débutant: "[je vais] tout de suite sur les [commentaires des]expérimentés, mais parce que l'expérimenté va me donner des outils qu'un débutant, [...] va p'têtre pas me donner des outils concrets qui puissent m'aider moi dans ma pratique en classe. ». En quête de bonnes pratiques, la PES va spontanément reproduire une situation d'enseignement descendant assimilant les expérimentés à des formateurs. Elle mobilise ici ses représentations hiérarchisées, en ce sens la majoration recherchée est d'ordre vertical. L'important degré de distance qu'elle sollicite entre elle-même et autrui conduit à l'échec du couplage et parallèlement à l'absence de majoration: " Voilà, par exemple là, elle [l'enseignante expérimentée] parle de petites choses simples qu'on peut très vite mettre en pratique et finalement elle les donne pas, alors c'est frustrant. » À l'inverse, Emilie, également lancée dans un processus d'enquête, visionne quatre commentaires de pairs débutants cherchant «des trucs qu['elle] n'fai[t] pas, qui ouvrent des idées». Elle parvient ici à faire coexister la proximité se projetant aisément dans l'activité du pair, avec la distance due à la diversité des regards: "En fait en voyant les autres c'est vrai qu'on a plus tendance à 
s'regarder soi-même, fin, à se regarder faire d'un point de vue extérieur ». Le fait de se voir comme un autre l'amène ici à problématiser son activité. En ce sens, le couplage majorant entrepris ici est d'ordre horizontal. Pour en revenir à Clothilde, ce n'est que lorsque qu'elle acceptera une majoration horizontale qu'elle s'installera dans une démarche réflexive: "C'est-à-dire que ça me donne pas une piste concrète mais maintenant ça me fait réfléchir sur justement la dépendance des élèvesj'ai p'têtre des élèves dans ma classe, moi qui sont dépendants [...] ça m'a permis de réfléchir sur moi, sur ma pratique mais c'est quelque chose, voilà, d'abstrait. ».

\section{III.3.2. Du point de vue des formateurs} enregistrements filmiques qui vont permettre un panel d'utilisations plus important que la seule observation directe en classe : «Le fait de réfléchir a posteriori, en revivant un présent qui n'est plus, permet une mise à distance de l'action qui la transforme en objet d'étude ». En se plaçant du point de vue de l'enseignant en formation, ils mettent en évidence notamment que la confrontation à des difficultés de pairs plutôt qu'aux siennes est sans doute plus favorable pour "faire ce pas de côté", partager un objet d'étude commun avec des collègues, se rassurer, travailler sur ses propres difficultés, envisager des perspectives de progrès réalistes et atteignables et prendre conscience de l'intérêt de mutualiser avec les autres.

En se plaçant du point de vue des enseignants filmés, les formateurs font preuve de vigilance par rapport à la production de jugements négatifs en totale extériorité qui ne respecteraient pas les conditions de confiance que les chercheurs-concepteurs ont dû créer pour obtenir leur participation à la production de ces ressources.

Ils font preuve d'empathie en adoptant ponctuellement leur place «Je les trouve quand même très courageux. Il n'est pas simple d'accepter l'idée d'être visionné et observé sur des "points faibles", même à distance! ». Cela les conduits à ériger et à partager un principe éthique à l'utilisation de film visant la protection des personnes « il faut pouvoir assurer que ceux qui la visionneront garderont un regard bienveillant ».

Pour finir, certains formateurs, sur la base des souvenirs de leur propre formation, accès sur les " préparations ", imaginent ce qu'ils auraient pu vivre de différents "Commej'aurais aimé avoir été formé avec Néopass@ction lorsque, il y a bien longtemps j'étais stagiaire... ». D'autres, enfin, qui n'exercent pas dans le domaine de l'enseignement, réussissent malgré tout à « voir en écho » à ces pratiques de visionnage de vidéo en formation leur propre pratique de formateur: "Je mets en place chaque année des ateliers de pratique théâtrale autour du "travail du clown" dans le cadre de l'unité d'enseignement "soins relationnels" avec les étudiants..."

Se mettre à la place de l'autre semble indispensable pour pouvoir appréhender et comprendre la situation vécue émotionnellement et intentionnellement de son point de vue : « comme nous n'avons aucune expérience immédiate de ce que les autres hommes ressentent, nous ne pouvons-nous faire une idée de la manière dont ils sont affectés, sinon en concevant ce que 
nous ressentirions nous-mêmes dans une pareille situation » (Jorland, 2004, p. 44). L'empathie envisagée comme une capacité à ressentir les émotions pour soi et pour autrui, à se glisser à la place de l'autre pour en comprendre les intentions tout en faisant la distinction entre soi et autrui constitue une disposition essentielle pour s'immerger dans une nouvelle situation et apprendre de cette situation fictionnelle.

\section{Conclusion}

Dans des conditions d'exploration de la plateforme différentes pour les PES et les formateurs (modalités et objectifs), l'utilité qui se dégage est à relier à la stimulation potentielle de différents processus impliqués dans la construction de l'expérience: une alternance entre différents points de vue, l'expression de jugements de valeur respectueux sur la manière d'agir d'autrui, des phénomènes d'empathie, une interrogation sur sa propre manière d'agir dans des situations similaires et la recherche de points de vue complémentaires. À travers la mise en parallèle de ces deux études, un certain nombre de tensions ont été également mises à jour: exploration libre vs exploration guidée; accès rapide à des réponses professionnelles vs construction de nouveaux problèmes; découverte de questions de métier inconnues vs ancrage dans sa propre pratique ; prise en compte du point de vue des acteurs vs jugement en extériorité. Ces tensions rendues visibles grâce à une mise en mots des acteurs adressée soit au chercheur soit au formateur sont autant de zones d'ombre qui restent à explorer aussi bien du point de la recherche que de l'accompagnement en formation.

\section{BIBLIOGRAPHIE}

Brunvand, S., Fishman, B. (2006), «Investigating the impact of the availability of the scaffolds on preservice teacher noticing and learning from video ", in Journal of Educational Technology Systems, $n^{\circ} 35(3)$.

Carre. P., Moisan. A., Poisson, D. (1997), L'autoformation. Paris : PUF.

Calandra, B., Puvirajah, A. (2011), « A framework for facilitating transformation in novice teachers using digital video », in Educational Technology, $n^{\circ} 51(2)$.

Durand, M. (2008), « Un programme de recherche technologique en formation des adultes : une approche enactive de l'activité humaine et l'accompagnement de son apprentissage/ développement ", in Education \& Didactique, $n^{\circ} 3(2)$.

Falzon, P. (2013), « Pour une ergonomie constructive », in Falzon P. (Ed.), Ergonomie constructive. Paris : PUF.

Flandrin, S., Leblanc, S., Muller, A. (2015), « Vidéoformation « orientée-activité » : quelles utilisations pour quels effets sur les enseignants? », in Raisons éducatives, $\mathrm{n}^{\circ} 19$. 
Gaudin, C., Chalies, S. (2012), «L'utilisation de la vidéo dans la formation professionnelle des enseignants novices : revue de littérature et zones potentielles d'étude ", in Revue Française de Pédagogie, $n^{\circ} 178$.

Jorland, G. (2004), « L'empathie, histoire d'un concept », in Berthoz, A. et Jorland, G. (Dir.), L'empathie. Paris : Editions Odile Jacob.

Leblanc, S., Seve, C. (2012), « Vidéo formation et construction de l'expérience professionnelle », in Recherche et Formation, $n^{\circ} 70$.

Lussi Borer, V., Muller, A. (2015), « Quel apport/usage du "voir "pour le "faire" en formation des enseignants du secondaire ", in Altet, M., Etienne, R., Paquay, L. et Perrenoud, P. (Eds.), Comment la formation des enseignants prend-elle en compte la réalité du travail enseignant et les prescriptions dont il fait l'objet dans le milieu scolaire? Bruxelles: De Boeck.

Paquelin, D. (2009), L'Appropriation des dispositifs numériques de formation: du prescrit aux usages. Paris : Harmattan.

Pastre, P. (2005), Apprendre par la simulation. De l'analyse du travail aux apprentissages professionnels. Toulouse : Octarès.

Prusak, K., Dye, B., Graham, C., Graser, S. (2010), « Reliability of pre-service physical education teachers'coding of teaching videos using studiocode analysis software ", in Journal of Technology and Teacher Education, $n^{\circ} 18(1)$.

Rabardel, P. (1995), Les hommes et les technologies. Paris : Armand Colin.

Ria, L., Leblanc, S. (2011), « Conception de la plateforme de formation Néopass@ction à partir d'un observatoire de l'activité des enseignants débutants : enjeux et processus ", in @ctivités, $n^{\circ} 8$ (2). http://www.activites.org/v8n2/v8n2.pdf [consulté le 12/04/2014].

Ria, L., Leblanc, S. (2012), « Professionnalisation assistée par vidéo : Les effets d'une navigation sur Néopass@ction », in Recherches et Educations, $n^{\circ} \%$.

Santagata, R. (2009), « Designing video-based professional development for mathematics teachers in low-performing schools ", in Journal of Teacher Education, $n^{\circ} 60(1)$.

Seidel, T., Blomberg, G., Renkel, A. (2013), « Instructional strategies for using video in teacher education », in Teaching and Teacher Education, $n^{\circ} 34$.

Theurau, J. (2004), Cours d'action : méthode élémentaire. Toulouse : Octarès.

Van Es, E., Sherin, M. (2008), « Mathematics teachers' "learning to notice" in the context of a video club ", in Teaching and Teacher Education, $n^{\circ} 24(2)$.

\section{NOTES}

1. Ces environnements sont censés d'une part, déclencher des processus mimétiques favorables

à l'immersion dans un environnement fictif qui possède certaines caractéristiques des environnements de travail et d'autre part, engendrer une activité différente de celle du travail en favorisant la création de décalage, le repérage de tensions, la délimitation de problèmes, l'imagination de solutions alternatives et en laissant du temps pour le faire. Les plateformes de vidéoformation (e.g. Néopass@ction), les Serious Games, les «micro-mondes » mais aussi les simulateurs de basse, moyenne ou haute-fidélité constituent des exemples de ces environnements utilisés dans différents contextes de formation professionnelle (santé, enseignement, management...). 
2. Ces vidéos sont prises en contexte réel mais lorsqu'elles sont visionnées en formation, elles le sont hors-contexte et déclenchent, chez les enseignants, une activité fictionnelle de «faire comme si » spontanée ou suscitée par le scénario de formation. C'est en ce sens qu'elles constituent des situations de simulation.

3. La plateforme de Néopass@ction est mentionnée dans le Bulletin officiel $n^{\circ} 2$ du 13 janvier 2011: Formation à la tenue de classe des professeurs et conseillers principaux d'éducation stagiaires et des personnels enseignants et d'éducation des établissements relevant du programme Clair: http://www.education.gouv.fr/cid54518/mene1001091c.html. Les thématiques développées sur Néopass@ction constituent des objets d'analyse professionnelle critiques pour des enseignants débutants car elles correspondent à leurs préoccupations d'entrée dans le métier (e.g. L'entrée en classe et la mise au travail, Faire parler les élèves à l'école élémentaire, Aider les élèves, Maternelle : rituel et consignes, Faire classe à cours double).

4. Les séances d'autoformation étudiées ne s'inséraient pas dans une formation avec des objectifs institués mais correspondaient à des pratiques en autodidacte.

5. L'activité silencieuse a été reconstruite en identifiant ce qui était pris en compte dans la situation par les acteurs, l'objet de leur engagement, leurs préoccupations, ce qu'ils faisaient, pensaient ou ressentaient, et ce qu'ils mobilisaient comme connaissances pour agir.

6. Un " environnement capacitant » est conçu pour favoriser la mise en œuvre effective des capacités des acteurs. Les concepteurs de ces environnements cherchent à déterminer les conditions favorables et les «facteurs de conversion» du potentiel d'action des acteurs en possibilité réelle.

\section{RÉSUMÉS}

Ce texte présente les principaux résultats de deux usages différents de la plateforme de vidéoformation Néopass@ction : a) l'un dans le cadre de navigations libres sur la plateforme par des professeurs-stagiaires en autoformation; b) l'autre dans le cadre d'un dispositif de formation à distance de formateurs d'enseignants. La mise en parallèle de ces deux études révèle un certain nombre de tensions en lien avec les visées de la formation professionnelle.

This text presents the main results of two different uses of the platform of online video training Néopass@ction: a) one use in self-study of novice teachers moving freely on the platform, b) the other use in a device of distance education for trainers of teachers. The paralleling of these two studies revealed a number of tensions related to the aims of vocational training.

\section{INDEX}

Keywords : Activity Analysis, Mimetic experience, Training of teachers, Video training Mots-clés : Analyse de l'activité, Expérience mimétique, Formation des enseignants, vidéo formation 


\section{AUTEURS}

\section{SERGE LEBLANC}

Professeur des universités, Faculté d'Éducation - Université de Montpellier, Laboratoire LIRDEF :

Faculté d'Éducation - Université de Montpellier et Université Paul Valéry Montpellier 3

\section{CÉLINE BLANES MAESTRE}

Doctorante, IFé-ENS Lyon, Laboratoire ECP 